\title{
Accreditation of SME Healthcare: Operators' Readiness
}

\author{
Nuraisyah Chua Abdullah' ${ }^{1}$, Herwina Rosnan², \\ Norzayana Yusof ${ }^{3}$
}

\author{
1 Faculty of Law, \\ ${ }^{2}$ Arshad Ayub Graduate Business School, \\ ${ }^{3}$ Faculty of Business and Management, \\ Universiti Teknologi MARA, Malaysia
}

nuraisyahc@yahoo.com, nuraisyah@salam.uitm.edu.my, herwinaros@gmail.com, norzayana.yusof@gmail.com

\begin{abstract}
SME healthcare includes the number of beds and other healthcare facilities that are not inpatient-based. While there are over 70 Malaysian healthcare centres that attract foreign patients, only thirteen are internationally accredited. Analysis from literature found that Malaysian SME healthcare providers are not ready for accreditation which partially due to financial and human resource constraints. Future research is called to gain empirical data on understanding the readiness, challenges and strategies to be implemented. The article is prominent in assisting the SME healthcare providers' behavioural change on the way towards accreditation for the growth of the medical tourism industry in Malaysia.

Keywords:Medical Tourism; SME Healthcare; Accreditation; Readiness

eISSN 2514-7528 @ 2019. The Authors. Published for AMER ABRA cE-Bs by e-International Publishing House, Ltd., UK. This is an open-access article under the CC BY-NC-ND license (http://creativecommons.org/licenses/bync-nd/4.0/). Peer-review under responsibility of AMER (Association of Malaysian Environment-Behaviour Researchers), ABRA (Association of Behavioural Researchers on Asians) and CE-Bs (Centre for EnvironmentBehaviour Studies), Faculty of Architecture, Planning \& Surveying, Universiti Teknologi MARA, Malaysia. https://doi.org/10.21834/jabs.v4i12.326
\end{abstract}




\subsection{Introduction}

Health and behaviour are the interconnected aspects of healthcare facilities due to the multidisciplined employees that work along in the institution as well as serving for patients from many walks of life (Setyowati, Harani, \& Falah, 2013). Beginning 1st January 2014, the SME Corp (2013), outlined that a new definition of services and other sectors that fall under SmallMedium Enterprise (SME) shall take effect. Accordingly, 'micro enterprises' are those that have sales turnover of less than RM300,000 or with employees of less than 5. Meanwhile, 'small enterprises' are those with sales turnover between RM300,000 and RM3 million or with employees between five to thirty. On the other hand, 'medium enterprises' are those with sales turnover between RM3 million to RM20 million or with employees between 30 to 75 people. While there is scarcity on the definition of hospital size, the traditional method of determining its size is based on the number of beds. This method is specifically for healthcare facilities that provide inpatient accommodations it may provide a good indicator of the hospital size. Hospitals can typically be divided into the following three main categories: large, medium, and small with various ways of determining the number of beds for each category. As an example, Ahmed, Rajagopalan, and Fuller (2015) posited that certain scholars classify small hospital are those with 200 beds and less, medium- 200 to 500 beds; and big- over 500 beds.

On the other hand, DNV-GL (2014), an accreditation agency in the US, defined SME healthcare facilities according to their level of electricity demand $(\mathrm{kW})$. Small healthcare facilities have less than $300 \mathrm{~kW}$ demand while medium-size healthcare facilities have an annual demand of 300 to $750 \mathrm{~kW}$. While there is no healthcare facilities under Malaysia Healthcare Travel Council (MHTC) is found to be listed on SME Corp to date, the present article approaches on SME Healthcare facilities which typically includes fertility centres, dental, clinics and ambulatory cares due to its relatively small serving capacity. Other healthcare facilities that are not in-patient based such as ambulatory surgery and medical centres, clinics, and dentists are considered to be small and medium healthcare facilities (Ahmed et al., 2015). Due to the rising demand for outpatient care (Samah, Ibrahim, Othman, \& Wahab, 2012), the present article finds it relevant to divert the focus on hospital accreditation to SME healthcare facilities which consist of clinics, dental clinics, treatment centres, spas and health supplies.

Meanwhile, healthcare accreditation was first introduced by the American College of Surgeons nearly a decade back in the United States (Smits, Supachutikul, \& Mate, 2014). As means to advance the quality of basic health service, accreditation is also adopted in lowand middle-income countries which Malaysia accounts for. Accordingly, the International Society for Quality in Healthcare's (ISQuA) defined accreditation as a public recognition by a healthcare accreditation body on a healthcare organisation's achievement of the accreditation standards via independent external peer assessment (Smits et al., 2014). In Malaysia, the Malaysian Society for Quality in Health (MSQH) stands as the accreditation organisation to the healthcare sector. It was established in 1999 by the Chief Secretary to the Government after being registered with Registry of Societies (ROS 470) as a legal entity. Wherein, healthcare facilities are invited to join as members and undergo the accreditation program according to their healthcare service category namely hospital, medical clinic, dental 
clinic. In general, MSQH caters for the accreditation of any healthcare facility defined by The Private Healthcare Facilities and Services Act 1998 which described it as any premises that provide healthcare services to one or more public member. Thus, MSQH provides survey program as means for the healthcare facility to reflect on their performance with regards to the relevant standards, as well as the accreditation program that aims for improving the service quality of the healthcare facility (Malaysian Society for Quality and Health, 2017).

It is also essential to note that 13 organisations consisting of hospitals and medical centres that are registered under the MHTC are $\mathrm{JCl}$-accredited(Joint Commission International, 2017). Nevertheless, dental and ambulatory care services are not included in the list. Accreditation plays an essential role in gaining prospectus medical tourists confidence before selecting a destination country. Hence, it is pivotal for the present paper to elaborate on the operators' readiness to pay their commitment to being granted the necessary accreditations continuously. Therefore, the present paper analyses the benefits of accreditation, the response and challenges that barricade the operators from being accredited as well as conceptualise on the potential undertakings to lift the barriers.

\subsection{Literature Review}

Having described the issue and problems in the medical tourism industry, the present section elaborates on the needs of ensuring that accreditation for SME healthcare operators is catered for. The discussion is then followed by the discussion on top accreditation bodies and their respective standards.

\subsection{Malaysian Government Attention on SME in tourism and medical tourism}

The national 2018 budget allocated a sum of RM2 billion to SME Tourism Fund to provide soft loans for tour operators with an interest subsidy of 2 per cent, and an additional RM1 billion to Tourism Infrastructure Development Fund as soft loans.RM500 million is also provided to develop and promote tourism by upgrading the infrastructure facilities and other tourism programmes. The tourism sector is deemed as one of the key sources of income to the nation's economy, and according to the World Tourism Organisation Report, Malaysia was ranked 12th regarding tourist arrivals in 2016(Ministry of Finance, 2017). With regard to medical tourism, the Government plans to allocate RM30 million to Malaysian Healthcare Travel Council (MHTC) to implement several initiatives among which is the provision of double tax deduction on expenses incurred to obtain certification from healthcare services accreditation bodies for dental and ambulatory services registered with MHTC.

Dental care standards relate to having qualified and competent dental professionals as required by licensing and accreditation, regulation of dental clinics, quality of dental care education, training of assistants, selection of equipment and supplies, and treating patients based on specific needs or personalized care. The Dental Clinic Standards was developed with collaboration between the Ministry of Health Malaysia (Oral Health Division), the Ministry of Defence Malaysia (Dental Service Division), and professional organisations representing the dental profession (Malaysian Society for Quality and Health, 2017). The objective of developing these standards is for the accreditation of dental clinics based on the core 
principles of safe dental practice, patient safety and the provision of high-quality services in dental clinics. Safety is an essential aspect to look at because healthcare buildings are often associated with moisture problem which could affect the ceiling, wall, floor and roof (Othman, Jaafar, Harun, \& Ibrahim, 2015)

\subsection{Prominent Healthcare Accreditation Bodies and Standards}

In Malaysia, the MSQH is responsible for providing accreditations to numerous types of healthcare facilities. MSQH covers for Hospital Accreditation Program (HAP) to assist hospitals in assessing their performance level against relevant national standards. The covered areas are i) Organisation and Management; ii) Human Resource Development and Management; iii) Policies and Procedures; iv) Facilities and Equipment; v) Quality Improvement Activities; and vi) Safety and Special Requirements. Apart from that, MSQH also caters for dental clinics (Malaysian Society for Quality and Health, 2017) which refers to all dental clinics and Dental Ambulatory Care Centres registered under the Private Healthcare Facilities and Services Act 1998 and public sector dental clinics, whose services include consultations, investigations, treatment and referrals.

Accordingly, the standards cover the following areas; i) access to Care; ii) facilities and Equipment; iii) human Resource; iv) Practice Addendum: Sedation; v) safety; vi) ethics; vii) Governance; viii) Quality Improvement Activities (Malaysian Society for Quality and Health, 2017). Apart from that, MSQH also serves as the Medical Clinic Accreditation Program (MCAP) to all 'free-standing' outpatient clinic services managed by medical practitioners in the public and private sectors inclusive of specialist clinics. The medical services include treatments, investigations and consultations. Thus, this program has established the Medical Clinic Standards in conjunction with the Ministry of Health, the Ministry of Defence and numerous other medical-related organisations. Therefore, the Standards were developed in view of ISQua Accreditation Federation Council principles and philosophies. Accordingly, the Standards cover for i) Access to care, ii) Practice; iii) Human Resource; iv) Safety; v) Ethical Practice; vi) Quality Improvement Activities (Malaysian Society for Quality and Health, 2017). On the other hand, Joint Commission International (2017) has also established the $\mathrm{JCl}$ Ambulatory Care in $\mathbf{2 0 1 5}$ for stand-alone medical, dental, and surgical facilities, dialysis facilities, diagnostic radiology centres, outpatient chronic care management facilities and acute care centres. Accordingly, the Standards first outline twelve requirements for operators among which include timely submission of information and data, and provision of complete and accurate information through all phases of the accreditation process. The Standards also delineated on the International Patient Safety Goals (IPSG) which aims to identify patients correctly, improve effective communication and reduce the risk of healthcare-associated infections. Apart from that, the Standards also elaborated on Access to Care and Continuity of Care (ACCC) to ensure referral and follow-ups, Patient and Family Rights (PFR), Assessment of Patients (AOP), Care of Patients (COP), Anaesthesia and Surgery Care (ASC), Medication Management and Use (MMU), Patient and Family Education (PFE), along with various other aspects that it covers. Thus, the comprehensive coverage that $\mathrm{JCl}$ and MSQH cover, only to name a few, resembles the pushing factor for healthcare facilities to improve its performance and quality while keeping up with the accreditation. Nevertheless, 
although $\mathrm{JCl}$ is one of the top accreditation bodies at international level, it is seen that many small and medium healthcare facilities are unable to attain its accreditation which reasons will be discussed in the following sections.

\subsection{Methodology}

Improvement of the healthcare quality has always been a continuous goal for the government which this paper is found to be corresponding with. In the $10^{\text {th }}$ Malaysia Plan, the government emphasised on the enforcement of healthcare service quality in both public and private sectors (Rani, Baharum, Akbar, \& Nawawi, 2015). This paper focuses on the response and readiness of SME healthcare in Malaysia to reciprocate to the requirements of the accreditation agencies as well as the strategies that follow. This area is chosen due to its lack of research in previous conferences as the discussion on medical practitioners' behaviour on accreditation in view of behavioural studies was never touched before.

Through analysis from journal articles, reports and newspaper articles, past literature discusses on the behaviour of SME healthcare operators towards the accreditation program, the challenges that they face and suggestions on the strategies that could be taken into account. Specifically, articles are selected based on several keywords such as "healthcare accreditation, "medical tourism", "SME healthcare", "challenges" and "strategies to implement accreditation" on ScienceDirect, Google Scholar and US National Library of Medicine. Emphasis is placed on publications between 2013 and 2018. Hence, discussions are presented in the form of critical analysis from past and present research. Information is derived from government reports and conceptual and primary data articles. Nevertheless, the authors acknowledge that the present paper lacks primary data which calls for future research to validate the presented arguments through empirical research.

\subsection{Results}

It is delineated that the problem of accreditation of SME healthcare lies on the operators' readiness and commitment to the accreditation programme. Several major findings of studies conducted in the field of accreditation specified that the healthcare workers responses about the accreditation are inadequate (Bahadori, Teymourzadeh, Ravangard, \& Alimohammadzadeh, 2016). Hence, research on understanding their views are still in infancy. Some researchers believe that accreditation is a tool assisting external stakeholders to find out how quality and safety are managed in an organisation (Bahadori et al., 2016). According to them, accreditation is an effective strategy for improving the team decisions and organisational performance and for assuring quality.

Furthermore, safety at healthcare centres stands as a space quality parameter, among several others, which constitutes the interior conditions of the centre (Samah, Ibrahim, \& Amir, 2013). Nevertheless, some critics also believe that accreditation leads to bureaucracy and spending much time and that it has limited impacts on improving patient care (Bahadori et al., 2016). However, findings also unveiled that there is an increasing trend on preparing 
for accreditation despite its vague effects on the organisation (Low, 2012; cited in Bahadori et al., 2016).

A study was also conducted on $307 \mathrm{PHC}$ healthcare professionals in Lebanon which found that majority of the respondents perceive accreditation as worthwhile and that it had a positive impact on the centres(El-Jardali et al., 2014). However, despite the great workshops conducted, only $78.2 \%$ indicated that the training they received was sufficient to run their accreditation duties. Regarding medical employees' behaviour, they are found to have to lack the quality and safety culture that would comply with accreditation. Besides, they also lack in-house collaboration and commitment to quality improvement. The time provided to learn and implement was inadequate that they require more time to understand and apply the accreditation concept.

Medical practitioners feel that accreditation is time-consuming especially with regard to the processing time and paperwork which is deemed might not necessarily lead to improved actual quality. This is said so because achieving accreditation may be merely on paperwork to meet the accreditation requirements. This tedious process also results in many senior practitioners leaving the workforce due to their inability to meet the technological advancement that medical practitioners are expected to keep up with and the meticulous documentation that is required. While word-of-mouth is still a strong medium of marketing to promote services in SME healthcare facilities and that the elder generation are prone to attend to healthcare facilities that they are familiar with (Chin, 2016), SME healthcare are doubted to posses to a strong view about healthcare accreditation.

In spite of the fact that patient satisfaction increased in accredited hospitals, the burden of administrative responsibilities to implement the accreditation process should be considered. As found in extant literature, it is seen that many healthcare centres are not prepared and inclined towards the accreditation program. This is due to several reasons such as that the concept is still new which would take time to get the personnel understood. What is even dire is that findings on Malaysian healthcare operators' readiness are scant which calls for future research to address this concern. Without having established work that reflects on the industry players' ability to commit to the accreditation program, it answers as to why Malaysian $\mathrm{JCl}$-accredited dental clinics and ambulatory care is low.

\subsection{Discussion}

Having presented the findings, the present section will delineate on the effects of accreditation, challenges to participate in the accreditation program and the strategies to improve on the implementation of the program.

\subsection{Effects of Accreditation on SME Healthcare}

Research on hospital accreditation program has been widely studied for many years. Nonetheless, there is still a lack of empirical findings on its implementation and effects, and the results are unstable (Bahadori et al., 2016). In addition to that, there is even scarce knowledge on the program, execution and effects of accreditation on SME healthcare. Nevertheless, the present article will still discuss some prevalent findings pertaining to the 
benefits and drawbacks of accreditation. In their study on primary healthcare (PHC), ElJardali et al. (2014) found that accreditation has led to quality improvement in several areas, particularly in documentation recording minutes of meetings, thoroughly completing medical records and documenting rules and regulations, as well as leadership and management. Accreditation also advances communication and teamwork between staff. Training also helped employees to perceive accreditation as an opportunity for career enhancement(ElJardali et al., 2014).

Accreditation also increases patient trust and satisfaction through the quality of services, reduced complaints, increase the inflow of patients from other regions (El-Jardali et al., 2014). Apart from that, accreditation also improves clinical services in an acute care hospital. It is unveiled that intervention rate on patients and the time spent on direct care is found to be higher on accredited acute care hospitals relative to the non-accredited (Um, Lyu, Lee, \& Park, 2018). Improved delivery of care and conducive healing environment do not only affect patient satisfaction and health (Ghazali \& Abbas, 2012), but it can be extended into strengthening the patients' relationship with their family members, thus creating stronger family units in the community (Ghani, Ainuddin, \& Dahlan, 2016). Apart from that, hope and trusts on healthcare providers are not only limited to patients but also the family members (Hashim \& Hussin, 2012) which strengthen for the needs to improve the quality of services in healthcare facilities.

Conclusively, it can be said that the accreditation program helps to materialise aspects of service quality. For instance, implementing procedures, policies, rules and regulations allows operators to practise their vision and mission. However, despite the general benefits of accreditation that stretches to different types of healthcare facilities, scholars have argued that the findings are inconsistent (Bahadori et al., 2016). Researchers in some studies have concluded that implementing accreditation offers limited impacts on improving patient care (Low, 2012; cited in Bahadori et al., 2016) with unclear organizational effectiveness, having many bureaucratic processes, time-consuming, does not carry significant impact on patient care, costly and unified assessors and surveyors (Yildiz \& Kaya, 2014).

\subsection{Challenges to Accreditation}

As Brubakk, Vist, Bukholm, Barach, and Tjomsland (2015) posited that there is scant evidence on the effectiveness of accreditation, the present article aspires to explore more on the challenges that SME healthcare operators face in attaining the desired accreditation. Therefore, it is found that commitment and support from management is crucial as facility directors reported facing many challenges in implementing accreditation. Resistance, especially among older staff, limited availability of physicians and specialists and the high turnover and workload, were reported to hinder the accreditation process(El-Jardali et al., 2014).

Moreover, the shortage of skilled human resources is also another concern as staffing shortages is a problem common to all types of healthcare organisations (El-Jardali et al., 2014). Furthermore, lack of communication, referral and follow-up and collaboration between centres are also another challenges to the implementation of the accreditation program. Moreover, El-Jardali et al. (2014) posited that limited financial resources were considered the 
major problem for implementing the new accreditation standards as they lack sufficient funding to meet the standards. Given this, employees also lack in education and training which would have allowed them to understand the importance and requirements needed for the accreditation. Centres also lack policies, procedures and planning which should be appropriately considered to provide a clear plan and direction for the staff.

In view of this, Zarifraftar and Aryankhesal (2016), who did a review on literature from 1960 to 2014, categorised these challenges into two namely, the programme and the organisational challenges. While the former illustrates the challenges pertaining to the ones that healthcare system face when attempting to have the accreditation programmes established, the latter focuses into the internal factors of healthcare organisations when they try to pass certain requirements for the accreditation. Therefore, Programme Challenges is further classified into three which are i) support of regulatory initiatives, ii) encouraging drivers, and iii) professional requirements. Hence, 'support of regulatory initiatives' may come in various forms including political support from the government and the presence of an accreditation body with a defined relationship with government. In particular, Zarifraftar and Aryankhesal (2016) denote that having an accreditation agency that is separated from the government body has the benefit of being less tied to the government policies and political changes. Nevertheless, government support is also deemed important to ensure safety and quality in healthcare organisations.

Moreover, the second challenge namely 'encouraging drivers' may happen through financial incentives which stand as among the strongest challenges to hospitals should incentives being cut down. Less participation by healthcare organisations in the accreditation process is also due to the lack of accreditation marketing by government and policymakers. Apart from that, the third challenge i.e. 'professional requirements' is also seen as one of the hindrances in the accreditation programme. This happens when there are less reliable survey practices through the validity of auditing by auditors, to name a few.

On the other hand, Zarifraftar and Aryankhesal (2016) explained that organisational challenges comprise of i) management \& organisation, ii) human resources, iii) financial \& facilities resources, and iv) quality improvements. Accordingly, 'management \& organisation' pertains to the challenges of knowledge, skills and commitment of hospitals managers with regard to the implementation of accreditation standards. Meanwhile, 'human resources' focuses on staff efficiency, creating motivation for the personnel that participate in this programme and providing education and technical assistance.

Further, organisational challenges also concern 'financial and facilities resources' specifically with the typical conditions of having many patients with limited physical space in the hospital, inadequate supplies, limited drugs and scarcity of resources that barricade the implementation of hospital accreditation. Apart from that, 'quality improvement' emphasises on issues on the lack of knowledge and skills about quality improvement (QI), integration and utilisation of the information system, participation of personnel and relationship with between sectors. Further, the balance between internal and external organisational objectives is also a subject of concern as hospitals are also to concentrate on improving the overall service quality while serving the patients rather than merely attaining certificates to satisfy the expected objectives. Therefore, a summary of the challenges in the implementation of 
accreditation in healthcare organisations is illustrated in Table 1 below.

Table 1: Challenges to the implementation of accreditation standards by healthcare organisations

\begin{tabular}{|l|l|}
\hline Program challenges \\
\hline \multirow{4}{*}{ Support of regulatory initiatives } & Legal support \\
\cline { 2 - 2 } & Independent/dependent accreditation agency \\
\cline { 2 - 2 } & Sustainable resourcing \\
\cline { 2 - 2 } & Voluntary or mandatory accreditation \\
\hline Encouraging drivers & Financial incentives \\
\cline { 2 - 2 } & Accreditation marketing \\
\hline Professional requirements & Perception of accreditation standards \\
\cline { 2 - 2 } & Standards development \\
\cline { 2 - 2 } & Surveying practices \\
\hline Organisation challenges & \\
\hline Management and organisation \\
\hline Human resources \\
\hline Financial \& facilities resources \\
\hline Quality improvement \\
\hline
\end{tabular}

(Source Zarifraftar \& Aryankhesal, 2016)

\subsection{Strategies for improving the implementation of accreditation}

Having elaborated that the accreditation bodies are providing the platforms for SME healthcare to improve their performance through the accreditation program, it is also revealed that the benefits of such program need further clarification. Apart from that, it is also unveiled that the operators do not possess strong readiness to pay commitment to accreditation programs. Therefore, the present section attempts to provide several strategies that SME healthcare could embark as a means to get them participated in the program.

Based on the findings on existing literature, it is seen that the employees working at the centres need continuous education and training. This is because accreditation is a new concept especially for people working in SME Healthcare centres, and some resistance is expected as with any managerial decisions that can affect day to day activities. As staff members are finding some difficulty with understanding the concept of accreditation, it is suggested to gradually integrate this concept into their daily work activities to obtain more extensive support. Staff should be trained not only on the importance of accreditation but also on the means to apply the standards, by changing their working culture. In view of this, the MSQH, as outlined before, provides numerous types of training and workshop where SME healthcare could participate in. As it is relatively feasible than conducting in-house training considering that healthcare facilities lack the expertise, the offered program should be utilised by the industry players as their effort to educate the employees. Training and time go along concurrently. Employees should be given ample time to under the training programs, to discuss with the team members in their respective departments, to strategies and later incrementally implement them.

As much as patients' needs are catered for, it is equally essential to consider and balance the needs of the healthcare operators (Kamali \& Abbas, 2012). The need for financial support is high for improving the implementation of accreditation. Limited financial resources were considered a major challenge to meeting the new accreditation standards. This support 
should come from the donors, the government and other stakeholders. As for Malaysia, the 2018 budget has planned to provide double tax deduction on expenses in attaining accreditation (Ministry of Finance, 2017). Although the effort is essential in encouraging foreign dental tourists to the country, it should be noted that there are still many dental clinics not registered to MHTC that need financial support let alone participating in the accreditation program. Unfortunately, these clinics are not included in the Budget.

Developing clear plans, policies and procedures, and guidelines were also viewed as a significant strategy to overcome the barriers to implementing the accreditation process. Respondents found that following clear plans and guidelines will allow them to understand better what is required of them and the goals and objectives they are aiming to achieve. It is also crucial to develop short- and long-term action plans for all the standards and work on the implementation of the healthcare facility. Apart from that, on MSQH's roles, there need to have diversified standards in all areas related to healthcare services and facilities. The present article finds it relevant considering that there are many SME healthcare centres with limited staffing and financial strength would find it difficult to keep up with the submissions and practices requirements. Hence, standards should also be categorised according to the size of the healthcare facility so that it can be appropriately translated into the firms' capacities. Therefore, an approach to designing the standards that cater to various types of healthcare facilities so that its implementation is relatively feasible should be taken into consideration.

Finally, conducting follow-up meetings and communication and collaboration with the accreditation team and among SME Healthcare centres, and hospitals are pivotal for sharing experiences on implantation of accreditation practices. In view of this, local experts should be involved in conducting the accreditation survey. Hence, the financial resources that would otherwise be used to employ foreign surveyors could be invested in improving the delivery of accreditation services. Constant follow-up is needed because SME healthcare centres cannot act on their own particularly that accreditation is a new concept for them. They need some follow-up to know whether the interventions they are making are effective and whether they are on the right track. Accordingly, involving leadership and management was also viewed as effective strategies to overcome challenges. Leadership support is very important in any quality improvement plan which would lead to the liable medical tourism industry. Responsible tourism practices are believed able to improve the quality of life of both service providers and medical tourist (Hanafiah, Azman, Jamaluddin, \& Aminuddin, 2016).

As an illustration of the above, the discussion is tabulated below to assist in the understanding of the strategies in order to overcome the barriers to SME healthcare's readiness for accreditation.

Table 2: Strategies to counter the barriers of SME healthcare readiness to accreditation

\begin{tabular}{|l|l|}
\hline Barriers to Accreditation & Strategies for Accreditation \\
\hline Unfamiliarity & Continuous education and training \\
\hline Difficulty in understanding & Gradual integration into daily work \\
\cline { 2 - 2 } & Training and workshop to amend work culture \\
\hline \multirow{2}{*}{ Limited staffing } & Ample time to strategise and gradually implement \\
\cline { 2 - 2 } & Develop policies, long-term and short-term plans \\
\hline
\end{tabular}


Financial constraints Financial support from donors, government and other stakeholders Constant follow-up

\subsection{Conclusion}

Conclusively, it is seen that many SME Healthcare centres need substantial support and technical assistance to build the capacity and systems to meet the standards for accreditation. This should not be too surprising given that many SME Healthcare facilities are having constraints regarding their staffing and finance. While the demand for efficient and productive improvements for healthcare facilities is high (Ngowtanasuwan \& Ruengtam, 2013) it should be noted that making the necessary changes will not be easy, as many involve changing practice culture particularly for areas related to shared decision making, extended practice hours, the expanded role of patients and families.

Furthermore, Zarifraftar and Aryankhesal (2016) concluded that prior to implementing the accreditation programs, governments are advised to provide the necessary basics such as support from accreditation programmes, sufficient funding, justifications on the obligation or voluntary nature of embarking on the programs, offering incentives for SME healthcare, as well as making the medical practitioners and the public aware of the accreditation programs. Attention should also be given on the SME healthcare organisations' needs and capacities in meeting the accreditation requirements. This should serve as the foundation of designing the standards. Moreover, the assessment process should be conducted regularly and without personal prejudice or in a manner that could harm confidence between all stakeholders (Zarifraftar \& Aryankhesal, 2016).

With the limited empirical findings on Malaysia's SME healthcare providers' readiness to embark on accreditation program, the present article suggests for future research endeavours to explore this matter. In healthcare centres where patients go through frequently and regularly, it offers a vast opportunity for healthcare scholars to conduct fieldwork and gain meaningful data from both the patients and service providers (Lawson, 2013). Not only would such research support the SME Healthcare in obtaining the recognition, but it is also likely to enhance their clinical and operational performance. Apart from that, it would also contribute to the usability namely effectiveness, efficiency, satisfaction (Haron, Hamida, \& Talib, 2012) of the accreditation program to SME healthcare service providers and patients. As a result, it opens more doors opportunity for SME healthcare to participate in the medical tourism industry program.

\section{Acknowledgement}

The authors would like to thank the Ministry of Higher Education Malaysia for the financial support through the Fundamental Research Grant Scheme (FRGS), File No: 600-RMI/FRGS $5 / 3$ (36/2015) and the Research Management Centre, Institute of Research Management \& Innovation (IRMI) Universiti Teknologi MARA, Shah Alam for managing the fund. 


\section{References}

Ahmed, T. M. F., Rajagopalan, P., \& Fuller, R. (2015). A Classification of Healthcare Facilities:Toward the Development of Energy Performance Benchmarks for Day Surgery Centers in Australia. HERD: Health Environments Research \& Design Journal, 8(4), 139-157. doi:10.1177/1937586715575910

Bahadori, M., Teymourzadeh, E., Ravangard, R., \& Alimohammadzadeh, K. (2016). Responses to the Criticisms about "The Accreditation of Hospitals in Iran". Iran J Public Health, 45(6), 840-842.

Brubakk, K., Vist, G. E., Bukholm, G., Barach, P., \& Tjomsland, O. (2015). A systematic review of hospital accreditation: the challenges of measuring complex intervention effects. BMC Health Services Research, 15, 280. doi:10.1186/s12913-015-0933-x

Chin, C. (2016). Keeping Our Patients. The Star Online. Retrieved from https://www.thestar.com.my/news/nation/2016/01/10/keeping-our-patients-malaysia-is-becoming-a-world-medicalhub-yet-malaysians-are-seeking-treatment-a/

DNV-GL. (2014). Market Sector Profile: Small and Medium Healthcare- Final

El-Jardali, F., Hemadeh, R., Jaafar, M., Sagherian, L., El-Skaff, R., Mdeihly, R., . . Ataya, N. (2014). The impact of accreditation of primary healthcare centers: successes, challenges and policy implications as perceived by healthcare providers and directors in Lebanon. BMC Health Services Research, 14(1), 86.

Ghani, S. N. A., Ainuddin, H. A., \& Dahlan, A. (2016). Quality of Life Amongst Family Caregivers of Older Persons with Terminal Illnesses. Procedia - Social and Behavioral Sciences, 234, 135-143. doi:https://doi.org/10.1016/j.sbspro.2016.10.228

Ghazali, R., \& Abbas, M. Y. (2012). Newly Built Public Paediatric Wards Increase Length of Stay (LOS)? In M. Y. Abbas, A. F. I. Bajunid, \& N. F. N. Azhari (Eds.), Ace-Bs 2012 Bangkok (Vol. 50, pp. 623-632). Amsterdam: Elsevier Science Bv.

Hanafiah, M. H., Azman, I., Jamaluddin, M. R., \& Aminuddin, N. (2016). Responsible Tourism Practices and Quality of Life: Perspective of Langkawi Island communities. In M. Y. Abbas, A. F. I. Bajunid, \& S. Thani (Eds.), AseanTurkey Asli Qol2015: Aicqol2015 (Vol. 222, pp. 406-413).

Haron, S. N., Hamida, M. Y., \& Talib, A. (2012). Towards Healthcare Service Quality: An Understanding of the Usability Concept in Healthcare Design. Procedia - Social and Behavioral Sciences, 42, 63-73. doi:https://doi.org/10.1016/j.sbspro.2012.04.167

Hashim, F., \& Hussin, R. (2012). Family Needs of Patient Admitted to Intensive Care Unit in a Public Hospital. In M. Y. Abbas, A. F. I. Bajunid, \& N. F. N. Azhari (Eds.), Asean Conference on Environment-Behaviour Studies (Vol. 36, pp. 103-111). Amsterdam: Elsevier Science Bv.

Joint Commission International. (2017). Achieve Accreditation. Retrieved from https://www.jointcommissioninternational.org/achieve-accreditation/

Kamali, N. J., \& Abbas, M. Y. (2012). Healing Environment: Enhancing Nurses' Performance through Proper Lighting Design. Procedia - Social and Behavioral Sciences, 35, 205-212. doi:https://doi.org/10.1016/j.sbspro.2012.02.080

Lawson, B. (2013). Design and the Evidence. Procedia - Social and Behavioral Sciences, 105, 30-37. doi:https://doi.org/10.1016/j.sbspro.2013.11.004 
Malaysian Society for Quality and Health. (2017). Education and Training Programmes. Retrieved from http://www.msqh.com.my/home/education-and-training-programmes

Ministry of Finance. (2017). 2018 Budget. Kuala Lumpur: Percetakan Nasional Malaysia Berhad.

Ngowtanasuwan, G., \& Ruengtam, P. (2013). Applied Simulation Model for Design of Improving Medical Record Area in Out-patient Department (OPD) of a Governmental Hospital. Procedia - Social and Behavioral Sciences, 101, 147-158. doi:https://doi.org/10.1016/j.sbspro.2013.07.188

Othman, N. L., Jaafar, M., Harun, W. M. W., \& Ibrahim, F. (2015). A Case Study on Moisture Problems and Building Defects. In M. Y. Abbas (Ed.), Asian Conference on Environment-Behaviour Studies (Vol. 170, pp. 27-36).

Rani, N. A. A., Baharum, M. R., Akbar, A. R. N., \& Nawawi, A. H. (2015). Perception of Maintenance Management Strategy on Healthcare Facilities. Procedia - Social and Behavioral Sciences, 170, 272-281. doi:https://doi.org/10.1016/j.sbspro.2015.01.037

Samah, Z. A., Ibrahim, N., \& Amir, J. S. (2013). Translating Quality Care Factors to Quality Space: Design Criteria for Outpatient Facility. Procedia - Social and Behavioral Sciences, 105, 265-272. doi:https://doi.org/10.1016/j.sbspro.2013.11.028

Samah, Z. A., Ibrahim, N., Othman, S., \& Wahab, M. H. A. (2012). Assessing Quality Design of Interiors: A case study of a Hospital Outpatient Unit in Malaysia. Procedia - Social and Behavioral Sciences, 35, 245-252. doi:https://doi.org/10.1016/j.sbspro.2012.02.085

Setyowati, E., Harani, A. R., \& Falah, Y. N. (2013). Green Building Design Concepts of Healthcare Facilities on the Orthopedic Hospital in the Tropics. In M. Y. Abbas (Ed.), Amer (Vol. 101, pp. 189-199). Amsterdam: Elsevier Science Bv.

SME Corp. (2013). New Definition of SMEs - An additional 8,120 establishments set to be classified as SMEs, bringing the share of SMEs to total establishments from $97.3 \%$ to $98.5 \%$. Retrieved from http://www.smecorp.gov.my/index.php/en/resources/2015-12-21-11-03-46/entrepre-news/162-entreprenews/tahun-2013/328-new-definition-of-smes-an-additional-8-120-establishments-set-to-be-classified-as-smesbringing-the-share-of-smes-to-total-establishments-from-97-3-to-98-5

Smits, H., Supachutikul, A., \& Mate, K. S. (2014). Hospital accreditation: lessons from low- and middle-income countries. Globalization and Health, 10, 65. doi:10.1186/s12992-014-0065-9

Um, M. H., Lyu, E. S., Lee, S. M., \& Park, Y. K. (2018). International hospital accreditation and clinical nutrition service in acute care hospitals in South Korea: results of a nationwide cross-sectional survey. Asia Pacific journal of clinical nutrition, 27(1), 158-166.

Yildiz, A., \& Kaya, S. (2014). Perceptions of nurses on the impact of accreditation on quality of care: A survey in a hospital in Turkey. Clinical Governance:. Clinical Governance: An International Journal, 19(2), 69-82.

Zarifraftar, M., \& Aryankhesal, A. (2016). Challenges of Implementation of Accreditation Standards for Health Care Systems and Organizations: A Systematic Review. Journal of Management Sciences, 2(3), 191-201. 